\title{
Exploration of the ethical dilemmas surrounding telephone follow-up clinics
}

\author{
in ENT Satabdhi Paul, Shadaba Ahmed
}

\section{INTRODUCTION}

Telemedicine refers to the provision of healthcare via electronic communicative devices such as telephones, when doctors and nurses are in different locations to their patients. ${ }^{1,2}$ These devices can be used to consult, triage or follow a patient up after they have been discharged postoperatively. ${ }^{3}$ The use of telecommunication to consult and triage is to assess the patient's complaint, categorise them based on the urgency of the call and then formulate a plan of action, for example either arrange a face-to-face consultation to allow appropriate examination or refer them on to a suitable specialist. ${ }^{3,4}$ Such practice has been developing over several years, and the purpose of it was to relieve pressure on doctors, decrease hospital activity and reduce unnecessary demands on other healthcare services. ${ }^{5}$ Several studies suggest that one half of the telephone consultations could be managed with advice over the telephone alone, making it an effective method of consultation. ${ }^{6,7,8}$

The use of teleconsultation in ENT was first suggested by Murthy et al. ${ }^{10}$ He proposed elimination of routine faceto-face follow up appointments in uncomplicated nasal surgery following a study, which found more than $75 \%$ of patients were satisfied post-surgery; many such findings have been reported since. ${ }^{10}$ A quality improvement survey exploring the use of telephone follow ups (TFU) in post-surgical ENT patients was conducted. ${ }^{9}$ The survey reported that the quality of ENT outpatient clinics had declined, as many patients were asymptomatic at the time of their appointment and so did not find it necessary to attend them. ${ }^{9}$ Therefore, this study was performed to explore an alternative to face-to-face appointments. TFU was found to be effective, with $90.5 \%$ of the patients not requiring face-to-face follow up. ${ }^{9}$

\section{AIM}

The purpose of this report is to explore the ethical implications when using TFU in post-surgical ENT patients. This report will use the four ethical principles plus scope tool to explore this method of doctor-patient communication and attempt to gauge whether it is ethically acceptable.

\section{BACKGROUND}

Septoplasty is a common ENT procedure that is performed to treat nasal obstruction caused by septal deviation. ${ }^{11}$ Distortion of the nasal septum which is the midline cartilaginous portion of the nose can be congenital or more commonly, caused by trauma. ${ }^{12}$ An article reported the indications for Septoplasty, highlighting the importance of the procedure not only due to its medical benefits but also in some cases, its cosmetic advantage. ${ }^{13}$ Septoplasty is extensively performed as it is considered a straightforward procedure with a high success rate; one study reported that $71 \%$ of the patients had shown significant clinical improvement and another reported more than two thirds of the patients had been relieved of long term nasal obstruction. ${ }^{14,15,16}$ Patients can be discharged on the same day following septoplasty and other ENT procedures such as grommet insertion. ${ }^{17}$

The four ethical principles plus scope is a tool that has been developed to serve as a guide when faced with ethical dilemmas in healthcare. ${ }^{18}$ Primarily, the role of the tool is to act as a framework for healthcare professionals to refer to when dealing with moral issues. It claims to be universal, providing common guidelines for healthcare ethics worldwide.

The first principle is respect for autonomy which refers to the patient's right to deliberate and make decisions about their own health. ${ }^{18}$ The second principle is beneficence or the act of doing 'good' and providing an overall benefit when they are under our care. This is directly associated with non-maleficence which is the third principle and refers to the act of doing no harm. One must weigh out risks and benefits when planning patient care and refrain from anything where the outcome will harm, rather than help the patient. ${ }^{18}$ Justice is the fourth principle which denotes the act of being fair and treating all patients equally and it has been further divided into three subsections - distributive justice, legal justice and rights based justice. ${ }^{18}$ Scope is the ability of the four principles to be applied to ethical scenarios; with regards to beneficence, for example, who are we obliged to help and when is it not unethical to not show a duty of beneficence? ${ }^{18}$ There may be situations when the principles cannot be integrated and this will be explored further.

\section{DISCUSSION}

Patient autonomy is the first principle and a crucial factor to consider during TFU. Visual prompts such as body positioning, facial expressions and eye contact aid faceto-face consultations and cannot be assessed with TFU. Visual prompts allow one to assess patient satisfaction. Patient satisfaction is an important indicator of patient autonomy. Reports suggest that patients are more likely to adhere to treatment if they are satisfied and feel autonomous. ${ }^{18}$ A study of instruments used to measure patient satisfaction was carried out to assess the patient's opinion on the quality of $\mathrm{TFU}^{3}$ The purpose was to reveal the level of autonomy patients believed they had, however findings were inconclusive. ${ }^{3}$ Studies also found that telephone consultations are generally shorter than face-to-face consultations. ${ }^{19}$ Consequences of a shorter TFU could mean a smaller chance for patients to convey their concerns, reduced satisfaction as a result, leading to reduced patient autonomy. ${ }^{19}$

Confidentiality has been an implicit expectation of all healthcare professionals in recent decades. It is especially 
relevant as it can reveal the level of autonomy one has been granted. By keeping information confidential a healthcare professional is respecting autonomy. Therefore, confidentiality is an important ethical consideration when applied to teleconsultation. Not only this, the culture of maintaining confidentiality makes autonomy applicable to this ethical scenario which, as discussed, is a requirement when using this tool.

Other ethical difficulties, such as patient identification, may arise when considering confidentiality with regards to TFU. During a traditional TFU, patient identity is confirmed by asking the full name, date of birth and first line of the address. Although it may be argued that these checks are sufficient in a face-to-face consultation, information can be falsified over the telephone, especially when you are unable to see who you are speaking to..$^{20,21}$ This raises not only ethical but also legal implications. A study investigating telephone consultations in general practice disclosed that patients were identified only by their name or simply voice recognition. ${ }^{22} \mathrm{~A}$ similar discovery was made whilst observing the ENT nurse-led TFU clinics in the University Hospitals of Morecambe Bay Trust (UHMBT). Parallel to the findings of the above study, basic information was requested before divulging confidential information. However, a pilot performed by an ENT ANP included a questionnaire suggesting patients were given a specific date and time of the TFU. ${ }^{23}$ This attempted to maintain confidentiality by ensuring the patient would be on the receiving end when the ANP called (Appendix). ${ }^{23}$

These findings violate the NHS Confidentiality Code of Practice which instructs all health care professionals to check all 'patients are who they say they are'; some practical suggestions were proposed to eliminate the problem. ${ }^{24}$ The emerging proposal was one of an individualised password on each patient record. ${ }^{24}$ This would be required from the patient before disclosing any information. Such policies can be considered by the ENT department to uphold confidentiality. All the same, the code of practice can be criticised for providing advice but no action plan, and trying to strictly abide by it could result in violating other ethical principles. For example, using the appointment time to confirm patient identity instead of addressing patient concerns would be a breach of beneficence..$^{25}$

Teleconsultations can be viewed to fulfil both beneficence and non-maleficence. TFU provides medical access for patients at a different geographical location to the healthcare facilities. The use of TFU post-operatively can benefit patients by allowing them to access the relevant healthcare professional for expert advice, and immediately if necessary. ${ }^{25} \mathrm{It}$ is beneficial for patients in rural areas with poor access to hospitals. In UHMBT, the responsibility of ENT patients alternates between Royal Lancaster Infirmary and Furness General Hospital each weekend. TFU post ENT surgery holds value here, as non-local patients can be assessed over the phone and transfer arrangements can be made. This allows physicians to act in the patient's best interest by preparing in case of postsurgical complications. Septoplasty has been reported to be low risk for post-operative complications; however a marginal risk of complications, such as septal haematoma remain ${ }^{26}$. The provision of TFU 6 weeks post-operatively demonstrates beneficence as patients are being checked for post-surgical complications.

Although less likely, a small percentage of patients who have a TFU require an out-patient appointment (OPA). ${ }^{9}$ Findings from the 6 month pilot study mentioned earlier are presented in table $1 .{ }^{23}$ The provision of TFU meant concerns could be escalated sooner, providing a net benefit. Conversely, TFU may be classed as a process that fails to uphold the act of beneficence and nonmaleficence. A physician is unable to see the patient when consulting over the telephone and consequently may miss a post-operative complication. This way, the patient may unintentionally be harmed, defying the principles of beneficence and non maleficence. Nonetheless, one may argue that patients with post-operative complication will have physical symptoms compelling them to consult a doctor even if this has been missed during the TFU; as stressed however, this is not always the case. TFU post ENT surgery has scope for application of the two ethical principles.

Justice can be divided into three categories; distributive, legal and rights based. Due to ongoing strains on NHS resources face-to-face consultations have become harder to access, and where appointments are available there is usually a waiting list. Distributive justice can be applied to TFU here; by organising nurse-led TFU clinics the pressure can be reduced for doctors. ${ }^{5}$ Not only does this allow post-surgical patients to be followed up quicker, but enables doctors to use their time where a more expert opinion is required. For instance, in the case of postoperative ENT patients, advanced nurse practitioners (ANP) can be trained to recognise problems and plan for optimal patient care. ${ }^{27,28}$ One study comparing a conventional follow up with nurse-led follow up showed more patients felt the need for a further appointment with the ENT specialist after the conventional follow up. Comparatively those who had been consulted by the nurse felt content with their meeting and did not require a further appointment. ${ }^{29}$

Contrary to the findings displayed above, patients who

\begin{tabular}{|l|l|l|l|l|}
\hline $\begin{array}{l}\text { Number of TFU } \\
\text { referrals post- } \\
\text { septoplasty }\end{array}$ & $\begin{array}{l}\text { Number receiving } \\
\text { TFU }\end{array}$ & $\begin{array}{l}\text { Number discharged } \\
\text { following first TFU }\end{array}$ & $\begin{array}{l}\text { Number referred } \\
\text { for second TFU }\end{array}$ & $\begin{array}{l}\text { Number referred } \\
\text { for an OPA }\end{array}$ \\
\hline 17 & $\begin{array}{l}13 \text { - not contactable } \\
\text { 1- Admitted }\end{array}$ & 9 & 2 & 2 \\
\hline
\end{tabular}

Table 1: Results from TFU conducted by ENT ANP in UHMBT over a 6 month period 
are followed up by ANP may feel that they have not had equal care in comparison to the patients who are reviewed by doctors. This will burden the NHS as patients will want additional appointments to see a doctor and increase the backlog of waiting lists. However, reports have been published indicating the complexity of patient satisfaction and the many factors that encompass it; the problem may not lie in the actual consulting, but a completely different matter such as the patient's personal and psychosocial issues. $^{30,31}$

TFU implicate rights based justice as some patients may argue that they have the right to be seen by a doctor immediately rather than having a TFU first or to speak to a doctor rather than a nurse. However, in UHMBT, post-operative patients are issued with ANP number post-discharge, at this point they may request to speak to or see a doctor instead and this would be respected. ${ }^{23}$ By informing the patient of the follow up procedure and giving them a chance to dispute it, their rights are being respected.

Legal justice comprises a significant ethical role in telephone consultations, due to the patient identity check dilemma. Through TFU healthcare professionals lack the ability to confirm patient identity and this can become legally unjust when patients falsify information over the telephone. ${ }^{25}$ Potential policies and their ethical flaws and benefits were discussed earlier in this paper. Malpractice is an ethico-legal concern regarding teleconsultations. In the USA complaints have been filed against health care professionals for providing an inadequate level of care during teleconsultations. ${ }^{32,} 33$ Such accusations warrant caution as patients may be dishonest about their care, to gain compensation. When applied to ENT consultations observed in UHMBT, the legal-justice scenario is plausible, especially with the lack of strategies in place to confirm patient identity. This has the potential for personal and confidential information to be disclosed, forming the basis of a legal encounter between the doctor and patient.

\section{CONCLUSION}

TFU have proven successful in specialties such as gastroenterology. ${ }^{9}$ A project on patients with inflammatory bowel disease showed the use of an annual nurse-led TFU resulted in satisfaction rates of up to $85 \% .^{9}$ From the literature review it is clear that these findings apply to ENT. TFU provide easy patient access to healthcare services and earlier identification of post-operative complications providing a net benefit for patients and avoiding harm. They relieve pressure on doctors and reduce unnecessary demands, allowing fair allocation of healthcare resources. Comparatively, TFU could be criticised for poor patient identification, poor satisfaction and confidentiality issues which could lead to legal implications.

A robust patient identification method could make TFU more ethical. The use of password protected access to patient notes could ensure correct patient identification so confidentiality is not breached. ${ }^{24}$ In UHMBT questionnaires were used to gauge patient satisfaction following TFU (table 1). However, this measure implicitly maintained confidentiality as patients were given a specific time, ensuring their availability when called. Not only this, patient autonomy was implied through the result of the questionnaires as patients were given a time and date, but also the opportunity to dispute and request and OPA. Such questionnaires should be used more widely due to the ethical benefits.

In conclusion, TFU has allowed healthcare to become more accessible. Patients are generally satisfied with this and their care, and if the ethical issues discussed above can be addressed, TFU can become an asset in all healthcare domains in coming years.

\section{REFERENCES}

1. Wootton. R. Telemedicine. BM7 2001;323:557-60.

2. Roine. R, Ohinmaa. A, Hailey. D. Assessing telemedicine: A systematic review of the literature. CMA7 2001;165(6):765-71.

3. Iseli. M.A, Kunz. R, Blozik. E. Instruments to assess patient satisfaction after teleconsultation and triage: a systematic review. Fournal of Patient Preference and Adherence 2014;8:893-907.

4. Lattimer. V, George. S. Nurse telephone triage in out-of-hours primary care: a pilot study. Primary Care Management 1996;6(5):3-6.

5. Bunn. F, Byrne. G, Kendall. S. The effects of telephone consultation and triage on healthcare use and patient satisfaction: a systematic review. $\mathrm{Br} 7 \mathrm{Gen}$ Pract 2005;55(521):956-61.

6. Christensen M. B, Olesen. F. Out-of-hours service in Denmark: Evaluation five years after reform. BM7 1998;316:1502-05.

7. Dale. J, Crouch. R, Lloyd. D. Primary care: nurse-led telephone triage and advice out of hours. Nurse Stand 1998;12(47):41-45.

8. Marsh. G. N, Horne. R. A, Channing. D. M. A study in telephone advice in managing out-of-hours calls. $\mathcal{F}$ $R$ Coll Gen Pract 1987;37:301-304.

9. Sooby. P, Kirkland. P. Use of nurse-led telephone follow-up as a sole method of assessing patients after nasal surgery. BM7 Qual improve Report 2015;4(1): doi: 10.1136/bmjquality.u208386.w3311.

10. Murthy. P, Mckerrow. W. S. Nasal septal surgery: is routine follow up necessary? 7 Laryngol Otol 1995;109:320-4.

11. Cukurova. I, Cetinkaya. E. A, Mercan. G. C, Demirhan. E. Retrospective analysis of 697 septoplasty surgery cases: packing versus trans-septal suturing method. Acta Otolaryngol Ital 2012;32(2):111-14.

12. Walikar. B. N, Rashinkar. S. M, Watwe. M. V, Anees. F, Kakkeri. S. A comparative study of Septoplasty with or without nasal packing. Indian 7 Otolaryngol Head Neck Surg 2011;63(3):247-48.

13. Saharia. P. S, Sinha. D. Septoplasty can change the shape of the nose. Indian 7 Otolaryngol Head Neck Surg 2013;65(2):220-25.

14. Siegel. N. S, Gliklich. R. E, Taghizadeh. F, Chang. Y. Outcomes of Septoplasty. Otolaryngol Head Neck Surg 2000;122(2):228-32. 
15. Samad. I, Stevens. H. E, Maloney. A. The efficacy of nasal septal surgery. 7 Otolaryngol 1992;21(2):88-91.

16. Stewart. M. G, Smith. T. L, Weaver. E. M, Witsell. D. L, Yueh. B, Hannley. M. T et al. Outcomes after nasal Septoplasty: results from the Nasal Obstruction Septoplasty Effectiveness (NOSE) study. Otolaryngol Head Neck Surg 2004;130(3):283-90.

17. Uppal. S, Lee. C, Mielcarek. M, Banks. P, Mackay. E, Coatesworth. A. A comparison of patient satisfaction with conventional and nurse-led outpatient followup after grommet insertion. Auris Nasus Larynx 2004;31(1):23-8.

18. Gillon. R. Medical ethics: four principles plus attention to scope. BMF 1994;309(6948):184-8.

19. Hewitt. H, Gafaranga. J, McKinstry. B. Comparison of face-to-face and telephone consultations in primary care: qualitative analysis. $\mathrm{Br} 7 \mathrm{Gen}$ Pract 2010;60(574):201-12.

20. Sokol. D, Car. J. Patient confidentiality and telephone consultations: time for a password. 7 Med Ethics 2006;32(12):688-89.

21. Nolan, F. (2001). Speaker identification evidence: its forms, limitations, and roles. In proceedings of the conference 'Law and Language: Prospect and Retrospect', Levi, Finnish Lapland.

22. Patel. H, Patel. M, Car. J. Telephone consultations in general practice: areas for improvement. Fournal of Telemedicine and Telecare 2005;11:265-266.

23. Ingham. T. (2015). The evaluation of a Telephone Follow Up clinic following uncomplicated nasal septoplasty. Unpublished manuscript.

24. Gov.uk (2003) 'Confidentiality: NHS Code of Practice.' $\mathrm{Pg}$ 7-45. Available at: [https://www.gov.uk/ government/publications/confidentiality-nhs-codeof-practice]. (Accessed on 15/04/2016).

25. Sokol. D, J. Car. Protecting patient confidentiality in telephone consultations in general practice. $\mathrm{Br} 7 \mathrm{Gen}$ Pract 2006;56(526):384-85.

26. Bloom. J. D, Kaplan. S. E, Bleier. B. S, Goldstein. S. A. Septoplasty complications: avoidance and management. Otolaryngol Clin 2009;42(3):463-81.

27. Gov.uk (1997) 'The New NHS' Available at: [https:// www.gov.uk/government/publications/the-new-nhs] (Accessed on 15/04/2016)

28. Lattimer V, George S, Thompson F, et al. Safety and effectiveness of nurse telephone consultation in outof-hours primary care: randomised controlled trial. BM7.1998;317:1054-59.

29. Uppal. S, Nadig. S, Mielcarek. M. W, Smith. L, Jose. J, Coatesworth. A. P. Patient satisfaction with conventional and nurse-led telephone follow-up after nasal septal surgery. Int 7 Clin Pract 2003;57(9):835-9.

30. Crow. R, Gage. H, Hampson. S, Hart. J, Kimber. A, Story. L et al. The measurement of satisfaction with healthcare: implications for practice from a systematic review of the literature. Health Technol Assess 2002;6(32):1-244.
31. Linder-Pelz.S,Struening.E. The multidimensionality of patient satisfaction with a clinic visit. 7 Community Health. 1985;10(1):42-54.

32. Terry. N.P. Cyber-malpractice: legal exposure for cyber-medicine. American Fournal of Law and Medicine 1999;25:327-66.

33. Spielberg. A. R. On call and online: socio-historical, legal and ethical implications of e-mail for the physician-patient relationship. Fournal of American Medical Association 1998;280:1353-9.

\section{APPENDIX}

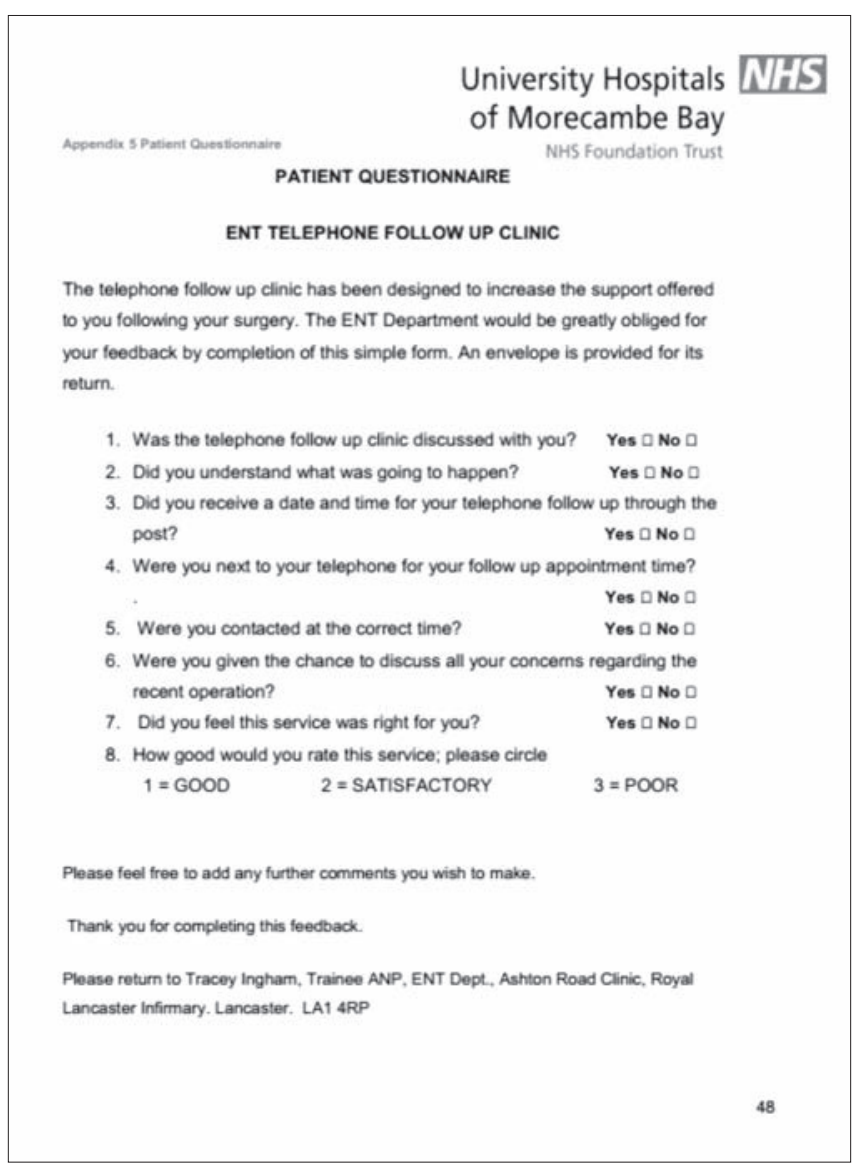

Questionnaire from the study carried out by ENT ANP in $\mathrm{UHMBT}^{23}$ 